\title{
Estudo Epidemiológico do Câncer Infantojuvenil no Hospital de Câncer de Cascavel Uopeccan entre os Anos 2000 e 2014
}

doi: https://doi.org/10.32635/2176-9745.RBC.2021v67n3.1224

\author{
Epidemiological Study of Childhood Cancer in Cascavel Cancer Hospital Uopeccan among 2000 and 2014 \\ Estudio Epidemiológico del Cáncer Infantil en el Hospital Oncológico de Cascavel Uopeccan entre 2000 y 2014
}

Bruna Cristina Longo'; Isabela Mangue Popiolek²; Natália Gomes do Vale³; Ana Lúcia Carrinho Ayroza Rangel; Maria Daniela Basso de Souza ${ }^{5}$

RESUMO

Introduçáo: Estudos epidemiológicos de base hospitalar sobre o câncer infantojuvenil são importantes para mostrar o perfil dos pacientes assistidos pelo serviço. Objetivo: Avaliar o perfil clínico-epidemiológico e a sobrevida de pacientes na faixa etária de 0-19 anos atendidos no Hospital do Câncer de Cascavel Uopeccan (2000-2014). Método: Estudo transversal com avaliação de prontuários para os seguintes desfechos: sexo, idade, cor/raça, outras patologias, histórico familiar de câncer, domicílio, tipo da neoplasia, estadiamento, tratamento, metástases, recidivas, situaçáo do paciente ao final da pesquisa. A estatística descritiva e os testes qui-quadrado e Kaplan-Meier foram aplicados. Resultados: Observou-se maior frequência para meninos (55,2\%), faixa etária de 1-4 anos (36,3\%), brancos (87\%), domicílio urbano $(81,6 \%)$, leucemia $(35,8 \%)$ e quimioterapia $(50,2 \%)$. Ocorreu metástase em $16,41 \%$ e recidiva em $22,38 \%$. Não havia relato de histórico familiar de câncer em $47 \%$ dos prontuários. Outras patologias foram negadas em 58,9\%. Ao final, 55,2\% estavam vivos e sem doença. Houve associação estatisticamente significava entre menores de 10 anos com tumores renais e neuroblastoma; maiores de 10 anos com linfomas e neoplasias epiteliais malignas; e entre a situação atual do paciente com metástase, recidivas e estadiamento. Conclusáo: Os pacientes analisados na presente pesquisa eram na maioria leucêmicos, do sexo masculino e faixa etária de 1-4 anos. A sobrevida global e a livre de doença foram, respectivamente, de 70,3\% e 71,63\%.

Palavras-chave: Neoplasias; Criança; Adolescente; Estudos Epidemiológicos; Análise de Sobrevida.

\section{ABSTRACT}

Introduction: Hospital-based epidemiological studies on childhood cancer are important to show the profile of patients cared by the service. Objective: To evaluate the characteristics of cancer patients aged 0-19 years at the Cascavel Cancer Hospital Uopeccan (2000-2014). Method: Cross-sectional study that evaluated medical charts for the following outcomes: gender, age, color/race, comorbidities, family history of cancer, household, cancer type, staging, treatment, metastasis, recurrences, patient's status at the end of study. Descriptive statistics, chi-square and Kaplan-Meier were applied. Results: Boys were more prevalent $(55.2 \%)$, age range from 1 to 4 years $(36.3 \%)$, White $(87 \%)$, urban household $(81.6 \%)$, with leukemia $(35.83 \%)$ and in chemotherapy $(50.2 \%)$. Metastasis occurred in $16.41 \%$ and recurrence in $22.38 \%$. There was no report of family history of cancer in $47 \%$ of the charts. Other pathologies were denied in $58.9 \%$. In the end, $55.2 \%$ were alive and disease-free. There was a statistically significant association between boys younger than 10 years old with renal tumors and neuroblastoma and older than 10 years with lymphomas and malignant epithelial neoplasms and between the current status of the patient with metastasis, relapses, and staging. Conclusion: The patients analyzed in this study were mostly leukemic, males and aged 1-4 years. Global and disease-free survival were, respectively, $70.3 \%$ and $71.63 \%$.

Key words: Neoplasms; Child; Adolescent; Epidemiologic Studies; Survival Analysis.

\section{RESUMEN}

Introducción: Los estudios epidemiológicos hospitalarios sobre cáncer infantil son importantes para mostrar el perfil de los pacientes atendidos por el servicio. Objetivo: Evaluar las características de los pacientes oncológicos de 0-19 años atendidos en el Hospital do Cáncer de Cascavel Uopeccan (2000-2014). Método: Estudio transversal que evaluó historias clínicas para los siguientes resultados: sexo, edad, color/raza, comorbilidades, antecedentes familiares de cáncer, domicilio, tipo de cáncer, estadificación, tratamiento, metástasis, recurrencias, situación del paciente al final de la investigación. Se aplicó estadística descriptiva, chi-cuadrado y Kaplan-Meier. Resultados: Fueron más prevalentes: niños (55,22\%), grupo de edad 1 a 4 años $(36,32 \%)$, blancos $(87,06 \%)$, hogares urbanos $(81,59 \%)$, leucemia $(35,83) \%)$ y quimioterapia $(50,25 \%)$. Hubo metástasis en $16,41 \%$ y recidiva en $22,38 \%$. No hubo informes de antecedentes familiares de cáncer en $47 \%$ de los pacientes. Se negaron comorbilidades en 58,91\%. Al final, 55,23\% estaban vivos y sin enfermedad. Hubo asociación estadística entre menores de 10 ańos con tumores renales y neuroblastoma; mayores 10 años con linfomas y neoplasias epiteliales malignas; e entre la situación del paciente con metástasis, recaídas y estadificación. Conclusión: Los pacientes analizados en esta investigación eran en su mayoría leucémicos, varones y de 1 a 4 ańos. La supervivencia global y libre de enfermedad fueron, respectivamente, 70,3\% y 71,63\%.

Palabras clave: Neoplasias; Niño; Adolescente; Estudios Epidemiológicos; Análisis de Supervivencia.

\footnotetext{
1-5Universidade Estadual do Oeste do Paraná (Unioeste). Centro de Ciências Biológicas e da Saúde. Cascavel (PR), Brasil.

'E-mail: bclongo@hotmail.com. Orcid iD: https://orcid.org/0000-0002-9498-3743

E-mail: popiolekisabela@hotmail.com. Orcid iD: https://orcid.org/0000-0003-3785-9188

3E-mail: natalia_gvw@hotmail.com. Orcid iD: https://orcid.org/0000-0003-3665-7496

${ }^{4}$ E-mail: alrangel2002@yahoo.com.br. Orcid iD: https://orcid.org/0000-0003-1080-358X

${ }^{5}$ E-mail: mdanibs@gmail.com. Orcid iD: https://orcid.org/0000-0002-7238-060X

Este estudo é parte da dissertação de Mestrado em Odontologia pela Unioeste de Bruna Cristina Longo, defendida em 14/2/2020.

Endereço para correspondência: Bruna Cristina Longo. Rua Cristóvão Colombo, 293 - Pioneiros Catarinenses. Cascavel (PR), Brasil. CEP 85805-510. E-mail: bclongo@hotmail.com
} 


\section{INTRODUÇÃO}

O câncer infantojuvenil é raro quando comparado ao do adulto $^{1}$ e, apesar de sua baixa prevalência, representa a principal causa de morte por doença nos países desenvolvidos em crianças maiores de $1 \mathrm{ano}^{2}$. No Brasil, é a primeira causa de morte por doença na faixa etária de 1 a 19 anos. Essas neoplasias representam um percentual médio de $1 \%$ a $4 \%$ do total de tumores malignos ${ }^{3}$. De acordo com o Instituto Nacional de Câncer José Alencar Gomes da Silva (INCA) ${ }^{4}$, para cada ano do triênio 20202022, estimam-se 8.460 novos casos, sendo 4.310 para o sexo masculino e 4.150 para o feminino.

Com isso, a avaliação de Regióes geográficas e temporais distintas contribuem para análise nas taxas de prevalência, incidência, sobrevida e mortalidade dessas doenças ${ }^{5}$. Dessa forma, as bases de dados hospitalares são necessárias para o conhecimento do perfil das neoplasias, melhora da assistência ao paciente, pesquisa clínica e auxílio na educação médica ${ }^{3}$. Considerando que até o presente momento dados sobre o câncer infantojuvenil na cidade de Cascavel-PR eram inexistentes, seu estudo e a análise dos dados tornam-se relevantes.

O câncer infantojuvenil apresenta grande variedade morfológica e características bastante peculiares quanto aos locais primários preferenciais de ocorrência, ao comportamento clínico e ao tipo histogenético ${ }^{6}$, afetando principalmente as células hematopoiéticas e mesenquimais ${ }^{6,7}$. Dessa forma, essas patologias são divididas de acordo com Classificaçáo Internacional do Câncer na Infância $(\mathrm{CICI})^{8}$, e os mais incidentes geralmente em crianças e adolescentes tanto no Brasil ${ }^{3,7,9-12}$ quanto em outras Regióes do mundo ${ }^{13-16}$ são as leucemias, os tumores do sistema nervoso central (SNC) e os linfomas.

Levando em conta as informaçóes apresentadas, o objetivo desta pesquisa foi analisar o perfil clínico-epidemiológico e a sobrevida de pacientes pediátricos do serviço de oncologia pediátrica do Hospital do Câncer de Cascavel Uopeccan entre 2000 e 2014.

\section{MÉTODO}

Estudo transversal com avaliação de prontuários (impresso e eletrônico) de crianças e adolescentes com até 19 anos de idade, diagnosticados e tratados para neoplasia maligna primária (de qualquer topografia), no Hospital de Câncer de Cascavel Uopeccan, entre os anos 2000 e 2014 (período necessário para cálculo de sobrevida).

O serviço de oncologia pediátrica é o único da Região Oeste do Paraná. Tem a área de cobertura (abrangência do hospital) de parte da $5^{\text {a }}$ regional de saúde (13 municípios) e abrangência completa, servindo como referência para as $7^{\mathrm{a}}, 8^{\mathrm{a}}, 9^{\mathrm{a}}, 10^{\mathrm{a}}, 11^{\mathrm{a}}, 12^{\mathrm{a}}$ e $20^{\mathrm{a}}$ regionais de saúde do Paraná.

Foram elegíveis todos os prontuários cujo diagnóstico foi confirmado por exames anatomopatológicos (histopatológicos e citopatológicos), hematológicos ou hemogramas, exploração cirúrgica, imagem, exame clínico ou qualquer outro meio ${ }^{7}$. Pacientes portadores de tumores benignos ou de comportamento incerto (se benignos ou malignos), que iniciaram o tratamento em outro hospital (laudo do diagnóstico ausente) ou transferidos antes do diagnóstico, foram excluídos da amostra.

A coleta de dados foi realizada entre outubro de 2018 e julho de 2019. As seguintes variáveis foram avaliadas: sexo, idade da detecção do câncer, raça/cor, outras patologias e procedência do paciente, histórico familiar de câncer e presença de outra patologia. Faixa etária e raça/cor basearam-se nas classificaçóes do INCA ${ }^{3}$ (menores de 1 ano; 1 a 4; 5 a 9; 10 a 14 e 15 e 19 anos) e do Instituto Brasileiro de Geografia e Estatística (IBGE) ${ }^{17}$ (branca, preta, parda, amarela e indígena), respectivamente.

O tipo de neoplasia seguiu a CICI-3, com base na Classificação Internacional de Doenças $(\mathrm{CID})^{8}$. Dados sobre estadiamento clínico para neoplasias sólidas, metástase no diagnóstico, recidiva e tratamento utilizado [quimioterapia $(\mathrm{QT})$; radioterapia $(\mathrm{RxT})$; cirurgia $(\mathrm{C})$; transplante de medula óssea (TMO)] e a situação do paciente no momento final da coleta dos dados da pesquisa (vivo sem doença; vivo com doença; óbito pela doença ou por outras causas) também foram coletados para análises de sobrevida (global e livre de doença). Considera-se sobrevida global o tempo em que o paciente permaneceu vivo após o diagnóstico da doença; e sobrevida livre de doença, o período em que náo se detectam sinais ou sintomas da doença após um tratamento curativo.

Os dados foram tabulados no programa Microsoft Excel (2010) e analisados para estatística descritiva no programa BioEstat 5.3 (Instituto Mamirauá) e apresentados em tabelas de contingência (frequências absoluta e relativa).

Empregou-se o teste qui-quadrado ou exato de Fisher, a fim de determinar a associação estatisticamente significante $(\mathrm{p} \leq 0,05)$ entre o tipo de neoplasia com sexo e faixa etária. Para essa análise, os tipos de neoplasias subclassificadas foram agrupados e comparados com os demais pesquisados. As faixas etárias foram divididas em menor e maior de 10 anos 9 . O mesmo teste foi empregado para análise de associação estatística significativa entre a situação atual do paciente com metástase, recidivas e estadiamento clínico. Nesse momento, foram considerados "vivos e sem doença" os que possuíam acompanhamento mínimo de cinco anos, após serem considerados curados. O teste qui-quadrado foi 
usado para análise de associação estatística significativa entre recidivas e estadiamento clínico.

Para os cálculos de sobrevida (programa Bioestat), foram utilizados o teste de Kaplan-Meier e o intervalo de confiança de $95 \%$. Consideraram-se as datas do início e do final do seguimento como a do diagnóstico do paciente e a da última informação no prontuário, respectivamente. Os pacientes que finalizaram o tratamento foram caracterizados como livres de doença; e os com informaçáo incerta ou ausente quanto à saúde ou doença, como doentes. As recidivas foram caracterizadas como ocorrência. Os que foram a óbito antes de término do tratamento ou que permaneciam em tratamento foram desconsiderados no cálculo da sobrevida livre de doença. O mês de julho de 2019 foi fixado como limite para o acompanhamento dos registros de sobrevida nos prontuários.

O estudo foi aprovado pelo Comitê de Ética em Pesquisa da Universidade Estadual do Oeste do Paraná (2.958.385) de acordo com a Resolução no. 466/12 do Conselho Nacional de Saúde ${ }^{18}$.

\section{RESULTADOS}

Dos 247 prontuários fornecidos pelo Hospital Uopeccan, 46 pacientes foram excluídos: recidivas (11); tumores benignos (6); recusa de tratamento pelo responsável (1); paciente não diagnosticado, transferência (8); início tratamento em outro hospital (13); falecimento antes do diagnóstico (1); prontuários incompletos (6). Assim, 201 foram incluídos com base nos critérios de inclusão e exclusão.

Considerando o total de casos, o sexo masculino foi o mais acometido ( $\mathrm{n}=111 ; 55,22 \%)$ (Tabela 1$)$. Entretanto, a avaliação individual da frequência entre sexos e cada tipo histogenético de neoplasia não mostrou associação estatisticamente significante ( $p>0,05)$.

Maior frequência do câncer infantojuvenil na faixa etária de $1-4$ anos $(n=73 ; 36,32 \%)$ (Tabela 1$)$ foi observada. Na comparação entre menores e maiores de 10 anos, detectou-se associação estatisticamente significante entre os maiores de 10 anos e os linfomas/

Tabela 1. Tipos de neoplasias malignas distribuídos por sexo e faixa etária $(n=201)$

\begin{tabular}{|c|c|c|c|c|c|c|c|c|c|c|c|c|c|c|}
\hline \multirow{3}{*}{\begin{tabular}{l}
\multicolumn{1}{c}{ Parâmetro } \\
\multicolumn{1}{c}{ Tipo de neoplasia } \\
Leucemias \\
Doenças mieloproliferativas e \\
mielodisplásicas
\end{tabular}} & \multicolumn{4}{|c|}{ Sexo } & \multicolumn{10}{|c|}{ Faixa etária } \\
\hline & \multicolumn{2}{|c|}{ Masculino } & \multicolumn{2}{|c|}{ Feminino } & \multicolumn{2}{|c|}{$<1$} & \multicolumn{2}{|c|}{$1-4$} & \multicolumn{2}{|c|}{$5-9$} & \multicolumn{2}{|c|}{$10-14$} & \multicolumn{2}{|c|}{$15-19$} \\
\hline & 42 & $20,9 \%$ & 30 & $14,9 \%$ & $*$ & * & 30 & $14,9 \%$ & 20 & $9,5 \%$ & 10 & $5,0 \%$ & 9 & $4,5 \%$ \\
\hline $\begin{array}{l}\text { Linfomas e neoplasias } \\
\text { reticuloendoteliais }\end{array}$ & 21 & $10,4 \%$ & 8 & $4,0 \%$ & * & $*$ & $*$ & * & 7 & $3,5 \%$ & 13 & $6,5 \%$ & 5 & $2,5 \%$ \\
\hline $\begin{array}{l}\text { SNC e diversas neoplasias } \\
\text { intracranianas e } \\
\text { intraespinhais }\end{array}$ & 5 & $2,5 \%$ & 6 & $3,0 \%$ & $*$ & $*$ & * & $*$ & 5 & $2,5 \%$ & $*$ & $*$ & $*$ & * \\
\hline $\begin{array}{l}\text { Neuroblastoma e outros } \\
\text { tumores de células nervosas } \\
\text { periféricas }\end{array}$ & 8 & $4,0 \%$ & 8 & $4,0 \%$ & $*$ & * & 9 & $4,5 \%$ & $*$ & $*$ & $*$ & $*$ & $*$ & * \\
\hline Retinoblastoma & $*$ & * & * & * & $*$ & * & $*$ & $*$ & $*$ & $*$ & $*$ & $*$ & $*$ & * \\
\hline Tumores renais & 6 & $3,0 \%$ & 11 & $5,5 \%$ & $*$ & $*$ & 8 & $4,0 \%$ & 7 & $3,5 \%$ & $*$ & $*$ & $*$ & $*$ \\
\hline Tumores hepáticos & * & $*$ & $*$ & $*$ & $*$ & * & $*$ & * & $*$ & * & $*$ & $*$ & $*$ & $*$ \\
\hline Tumores de osso malignos & $*$ & $*$ & 5 & $2,5 \%$ & $*$ & $*$ & $*$ & $*$ & $*$ & $*$ & $*$ & $*$ & $*$ & * \\
\hline $\begin{array}{l}\text { Tecidos moles e outros } \\
\text { sarcomas extraósseos }\end{array}$ & 10 & $5,0 \%$ & 8 & $4,0 \%$ & $*$ & $*$ & 8 & $4,0 \%$ & $*$ & $*$ & $*$ & * & $*$ & * \\
\hline $\begin{array}{l}\text { Tumores de células } \\
\text { germinativas, tumores } \\
\text { trofoblásticos e neoplasias em } \\
\text { gônadas }\end{array}$ & 5 & $2,5 \%$ & 5 & $2,5 \%$ & $*$ & $*$ & $*$ & $*$ & $*$ & $*$ & $*$ & $*$ & $*$ & $*$ \\
\hline $\begin{array}{l}\text { Outras neoplasias epiteliais } \\
\text { malignas e melanomas } \\
\text { malignos }\end{array}$ & 8 & $4,0 \%$ & 7 & $3,5 \%$ & $*$ & $*$ & $*$ & * & $*$ & $*$ & 6 & $3,0 \%$ & $*$ & * \\
\hline $\begin{array}{l}\text { Neoplasias malignas } \\
\text { diferentes e não especificadas }\end{array}$ & $*$ & $*$ & $*$ & $*$ & $*$ & $*$ & * & $*$ & $*$ & $*$ & $*$ & $*$ & $*$ & $*$ \\
\hline Total & 111 & $55,2 \%$ & 90 & $44,8 \%$ & 14 & $6,9 \%$ & 73 & $36,3 \%$ & 51 & $25,3 \%$ & 38 & $18,9 \%$ & 25 & $12,4 \%$ \\
\hline
\end{tabular}

Legenda: SNC: sistema nervoso central.

$\left(^{*}\right)$ valores menores do que 5 pacientes por grupo. 
neoplasias reticuloendoteliais $(\mathrm{p}=0,0003)$ e outras neoplasias epiteliais malignas/melanomas $(\mathrm{p}=0,003)$. Com os menores de 10 anos, por sua vez, observou-se associaçáo estatisticamente significante com os tumores renais $(\mathrm{p}=0,01)$ e neuroblastoma/outros tumores de células nervosas periféricas $(\mathrm{p}=0,01)$.

Entre os tipos de neoplasias, a leucemia $(n=72$; $35,83 \%$ ) foi mais frequente, em particular a linfoide ( $\mathrm{n}=63 ; 87,05 \%$ das leucemias). Em seguida, os linfomas $(\mathrm{n}=29 ; 14,42 \%)$, particularmente o Hodgkin $(\mathrm{n}=19$; $65,51 \%$ dos linfomas) e os tumores renais (nefroblastomas ou tumor de Wilms) (n=17; 8,46\%) (Tabela 1).

A condição sociodemográfica dos pacientes, tratamentos e situaçáo ao final da coleta de dados são descritos a seguir. A raça/cor branca foi a mais frequente $(87 \%)$. Mais de três quartos residiam em área urbana $(81,6 \%)$. Outras patologias foram encontradas em 128 prontuários $(62,7 \%)$. Quatro crianças (2\%) eram sindrômicas, sendo dois portadores da síndrome de Down e leucemia, um com síndrome de Bloom e leucemia e outro com tumor de Wilms (nefroblastoma) e síndrome de Beckwitch Wiedemann. Registros do histórico familiar de câncer foram encontrados em 108 (53,7\%) prontuários; alguns deles relataram mais de um familiar acometido pela doença e pacientes com mais de uma patologia, o que justifica o somatório superior a $100 \%$ nessas variáveis. A maior parte realizou QT isolada $(50,2 \%)$ ou associada à outra terapia (40,3\%). A situação do paciente foi checada e tabulada ao final do período de coleta de dados: $55,2 \%$ deles estavam vivos sem doença. Não havia informação acerca do abandono de tratamento e da perda de seguimento.

O estadiamento clínico dos tumores sólidos está representado na Tabela 3. Essa informação, que deveria constar em 129 prontuários, inexistia em 48 deles, sendo assim o número de casos com estadiamento determinado foi de 81 pacientes.

Considerando um período de cinco anos, a sobrevida global $(n=201)$ e a livre de doença $(n=130)$ foram, respectivamente, de 70,3\% (Gráfico 1) e 71,63\% (Gráfico 2 ), pois os demais pacientes permaneciam em tratamento ou foram a óbito.

A presença de metástase no momento do diagnóstico foi observada em 33 (16,41\%) pacientes e 45 (22,38\%) tiveram recidiva da doença. A situação atual foi associada a recidivas $(\mathrm{p}=0,0004)$ e a metástases $(\mathrm{p}=0,01)$ (qui-quadrado/exato de Fisher) ( $\mathrm{n}=108)$.

Apenas 81 pacientes tiveram o estadiamento clínico determinado. O teste qui-quadrado náo detectou associação significativa entre essa variável e as recidivas $(\mathrm{p}=0,88)$. Entretanto, o teste exato de Fisher evidenciou associação significativa entre a situação do paciente
Tabela 2. Perfil dos pacientes com relação à condição sociodemográfica, tratamentos e situação ao final da coleta de dados

\begin{tabular}{lcc}
\multicolumn{1}{c}{ Parâmetro } & N & $\%$ \\
\hline Raça/Cor da pele & & \\
Branca & 175 & $87 \%$ \\
Negra & 13 & $6,5 \%$ \\
Parda & 10 & $5 \%$ \\
Amarela & 2 & $1 \%$ \\
Indígena & 1 & $0,5 \%$ \\
Local de residência & & \\
Urbana & 164 & $81,6 \%$ \\
Rural & 37 & $18,4 \%$ \\
Presença de outras patologias & & \\
Asma & 1 & $0,5 \%$ \\
Bronquite & 2 & $1 \%$ \\
Diabetes & 2 & $1 \%$ \\
Esclerose tuberosa & 1 & $0,5 \%$ \\
Intolerância à lactose & 1 & $0,5 \%$ \\
Paralisia infantil & 1 & $0,5 \%$ \\
Lesão neurológica não & 1 & $0,5 \%$ \\
especificada & 1 & $0,5 \%$ \\
Lesão cardíaca não especificada & 18 & $57,7 \%$ \\
Negaram & &
\end{tabular}

\section{Histórico familiar de câncer}

$\begin{array}{lcc}\text { Avós } & 34 & 16,9 \% \\ \text { Tios } & 14 & 6,97 \% \\ \text { Bisavós } & 7 & 3,5 \% \\ \text { Pais } & 5 & 2,5 \% \\ \text { Primos } & 5 & 2,5 \% \\ \text { Irmãos } & 5 & 2,5 \% \\ \text { Negaram } & 56 & 27,9 \%\end{array}$

\section{Tipo de tratamento}

Quimioterapia 101

Quimioterapia + cirurgia

$10150,2 \%$

Quimioterapia + radioterapia

$35 \quad 17,4 \%$

$24 \quad 11,9 \%$

Quimioterapia + radioterapia + cirurgia

$115,5 \%$

Quimioterapia + transplante de medula óssea

$84 \%$

Quimioterapia + radioterapia + transplante de medula óssea

Cirurgia

Radioterapia

Cirurgia + radioterapia

Não realizaram

Situação final do paciente

Vivos sem doença

$11155,2 \%$

Vivos com doença

$30 \quad 14,9 \%$

Óbito pela doença 
Tabela 3. Tipo de neoplasia sólida de acordo com o estadiamento clínico ( $n=129)$

\begin{tabular}{|c|c|c|c|c|c|c|}
\hline \multirow{3}{*}{ Tipo de neoplasia } & \multicolumn{5}{|c|}{ Estadiamento clínico } & \multirow{2}{*}{ Total } \\
\hline & I & II & III & IV & SR & \\
\hline & N $\%$ & N $\%$ & N $\%$ & N $\%$ & N \% & $\mathbf{N}$ \\
\hline
\end{tabular}

Linfomas e neoplasias reticuloendoteliais

Linfomas de Hodgkin

Linfomas não Hodgkin (exceto linfoma de Burkitt)

$\begin{array}{llllllllllll}2 & 1,5 & 9 & 7 & 3 & 2,3 & 5 & 3,9 & 0 & 0 & 19 & 14,8\end{array}$

Linfoma de Burkitt

$\begin{array}{cccccccccccc}0 & 0 & 0 & 0 & 3 & 2,3 & 1 & 0,8 & 2 & 1,5 & 6 & 4,6 \\ 1 & 0,8 & 0 & 0 & 0 & 0 & 0 & 0 & 3 & 2,3 & 4 & 3,1\end{array}$

SNC e diversas neoplasias intracranianas e intraespinhais

\begin{tabular}{|c|c|c|c|c|c|c|c|c|c|c|c|}
\hline Astrocitomas & 0 & 0 & 0 & 0 & 0 & 0 & 0 & 0 & 1 & 0,8 & 1 \\
\hline Tumores embrionários intracranianos e intraespinhais & 1 & 0,8 & 0 & 0 & 0 & 0 & 1 & 0,8 & 3 & 2,3 & 5 \\
\hline Outras neoplasias intracranianas e intraespinhais & 0 & 0 & 0 & 0 & 0 & 0 & 0 & 0 & 1 & 0,8 & 1 \\
\hline $\begin{array}{l}\text { Neoplasmas intracranianos e intrapsíquicos não } \\
\text { especificados }\end{array}$ & 0 & 0 & 0 & 0 & 1 & 0,8 & 0 & 0 & 3 & 2,3 & 4 \\
\hline
\end{tabular}

Neuroblastoma e outros tumores de células nervosas periféricas

Neuroblastoma e ganglioneuroblastoma

Retinoblastoma

$\begin{array}{cccccccccccc}1 & 0,8 & 0 & 0 & 1 & 0,8 & 9 & 7 & 5 & 3,9 & 16 & 12,4 \\ 0 & 0 & 0 & 0 & 0 & 0 & 0 & 0 & 1 & 0,8 & 1 & 0,8\end{array}$

Tumores renais

Nefroblastoma e outros tumores renais não pélvicos

$\begin{array}{llllllllllll}5 & 3,9 & 4 & 3,1 & 0 & 0 & 6 & 4,6 & 2 & 1,5 & 17 & 13,2\end{array}$

Tumores hepáticos

Hepatoblastoma

$\begin{array}{llllllllllll}0 & 0 & 0 & 0 & 0 & 0 & 2 & 1,5 & 2 & 1,5 & 4 & 3,1\end{array}$

Tumores de osso malignos

Osteossarcomas

Condrossarcomas

$\begin{array}{llllllllllll}0 & 0 & 0 & 0 & 0 & 0 & 1 & 0,8 & 5 & 3,9 & 6 & 4,6\end{array}$

Tecidos moles e outros sarcomas extraósseos

Rabdomiossarcomas

Outros sarcomas de tecidos moles especificados

$\begin{array}{llllllllllll}1 & 0,8 & 3 & 2,3 & 3 & 2,3 & 1 & 0,8 & 6 & 4,6 & 14 & 10,9 \\ 0 & 0 & 0 & 0 & 1 & 0,8 & 1 & 0,8 & 1 & 0,8 & 3 & 2,3 \\ 0 & 0 & 0 & 0 & 0 & 0 & 0 & 0 & 1 & 0,8 & 1 & 0,8\end{array}$

Sarcomas não especificados de tecidos moles

Tumores de células germinativas, tumores trofoblásticos e neoplasias em gônadas

Tumores de células germinativas intracranianas e intraespinhais

Tumores malignos de células germinativas gonadais

$$
\begin{array}{rrrrrrrrrrrr}
0 & 0 & 0 & 0 & 0 & 0 & 0 & 0 & 2 & 1,5 & 2 & 1,5 \\
0 & 0 & 3 & 2,3 & 2 & 1,5 & 1 & 0,8 & 2 & 1,5 & 8 & 6,2
\end{array}
$$

\begin{tabular}{|c|c|c|c|c|c|c|c|c|c|c|c|c|}
\hline Carcinomas adrenocorticais & 0 & 0 & 2 & 1,5 & 0 & 0 & 1 & 0,8 & 4 & 3,1 & 7 & 5,4 \\
\hline Carcinomas de tireoide & 1 & 0,8 & 0 & 0 & 1 & 0,8 & 0 & 0 & 0 & 0 & 2 & 1,5 \\
\hline Melanomas & 2 & 1,5 & 0 & 0 & 0 & 0 & 0 & 0 & 1 & 0,8 & 3 & 2,3 \\
\hline Outros carcinomas não especificados & 1 & 0,8 & 0 & 0 & 0 & 0 & 1 & 0,8 & 1 & 0,8 & 3 & 2,3 \\
\hline \multicolumn{13}{|c|}{ Neoplasias malignas diferentes e não especificadas } \\
\hline Outros tumores malignos especificados & 0 & 0 & 0 & 0 & 0 & 0 & 0 & 0 & 1 & 0,8 & 1 & 0,8 \\
\hline Total & 15 & 11,6 & 21 & 16,3 & 15 & 11,6 & 30 & 23,4 & 48 & 37,1 & 129 & 100 \\
\hline
\end{tabular}

Outras neoplasias epiteliais malignas e melanomas malignos

Legenda: SR: sem relato. 


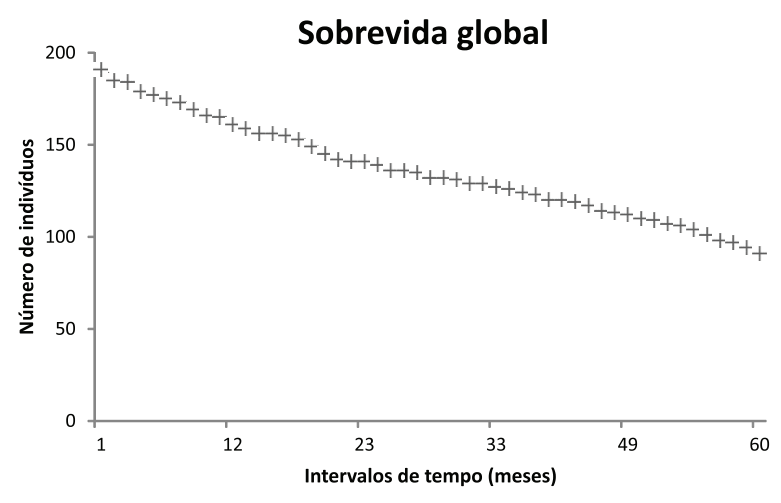

Gráfico 1. Sobrevida global $(n=201)$

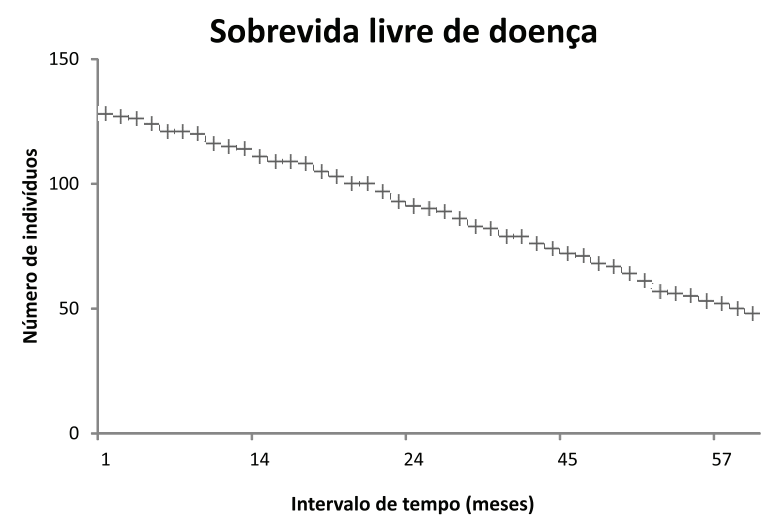

Gráfico 2. Sobrevida livre de doença $(n=130)$

e o estadiamento clínico $(\mathrm{p}=0,0004)$, para o qual consideraram-se 46 pacientes.

\section{DISCUSSÃO}

Este estudo analisou o perfil clínico-epidemiológico e a sobrevida de pacientes pediátricos atendidos no serviço de oncologia pediátrica do Hospital do Câncer de Cascavel Uopeccan, localizado na Regiáo Oeste do Estado do Paraná. Este presta assistência para a população da cidade de Cascavel e contempla sete regionais de saúde.

Conhecer o perfil do câncer infantojuvenil no Brasil é relevante para ampliação de ações efetivas para o controle da doença e planejamento ${ }^{\mathrm{d}}$ e serviços3. Dessa forma, os meninos $(55,2 \%)$ e a cor/raça branca (87\%) (Tabelas 1 e 2 ) foram predominantes na população estudada. A ausência de associação estatística significativa entre o sexo e o tipo de neoplasia observada pode ser explicada pelo pequeno número de indivíduos estudados, bem como pela diferença percentual entre os sexos. $\mathrm{O}$ resultado do perfil racial era esperado, pois a cor/raça branca é maioria no Estado do $\operatorname{Par}^{\text {aná }}(65,5 \%) 17$.

Quanto à idade, a faixa etária de 1 a 4 anos foi a mais acometida (Tabela 1) e observou-se associaçáo estatisticamente significativa entre os tipos de neoplasia e a faixa etária. Esperava-se que o Tumor de Wilms e o neuroblastoma fossem mais frequentes nos menores de 10 anos, já que ambos são tumores embrionários comuns em crianças menores de 5 anos e originam-se de células primordiais que sofreram mutaçóes espontâneas e não mutaçóes adquiridas por açôes ${ }^{a}$ mbientais13. Após os 10 anos, em geral, eles tendem a desaparecer e outros cânceres se tornam mais frequentes (linfomas, carcinomas, tumores de células germinativas e tumores ósseos) ${ }^{9,13}$. Os resultados demonstraram predileção por maiores de 10 anos para os linfomas/neoplasias reticuloendoteliais e neoplasias epiteliais malignas/melanomas. Os linfomas (principalmente de Hodgkin), melanomas e carcinomas são mais comuns em adolescentes ${ }^{9,13}$.

A leucemia foi a neoplasia mais frequente, em particular a do tipo linfoide (87\% delas) (Tabela 1). A literatura demonstra que a leucemia linfoide aguda (LLA) é mais comum em crianças, correspondendo a $75 \%$ a $80 \%$ do total de casos de leucemia'. O linfoma do tipo Hodgkin, que afeta mais os adolescentes ${ }^{13}$, atingiu mais $\mathrm{da}$ metade dos pacientes (Tabela 1) e pode ser relacionado ao número de linfomas encontrado nos maiores de 10 anos (Tabela 1). Apesar da possibilidade de um diagnóstico tardio ter atuado como fator de confusão nesta análise, este foi atenuado pela amplitude da divisão entre menores e maiores de 10 anos. A terceira neoplasia mais frequente foi o tumor de Wilms (Tabela 1) que, em geral, é a neoplasia renal mais comum na infância. Os tumores de SNC, por sua vez, foram o sexto em frequência (Tabela 2).

A maior parte da população avaliada no presente estudo era residente de área urbana (Tabela 2). Observou-se, ainda, a escassez de informação nos prontuários acerca do histórico familiar de câncer, bem como a baixa frequência de pacientes sindrômicos com a doença (2\%). Um estudo demonstrou que de fato esses pacientes sindrômicos representam uma pequena parcela dos casos de câncer infantojuvenil ${ }^{19}$.

O diagnóstico da neoplasia e o estágio da doença determinam a terapêutica a ser aplicada ${ }^{20}$, e a maioria dos pacientes do presente estudo passou por QT isolada ou associada à outra modalidade (Tabela 2). Ao inibir a proliferação celular de células tumorais (nas em fase mitótica, preferencialmente), os agentes quimioterápicos acabam por atuar nas não tumorais também. Assim, as crianças (em desenvolvimento e crescimento celular) são particularmente propensas a efeitos colaterais ${ }^{21}$, o que justifica estudos sobre o tema.

Há relatos sobre a evolução dos tratamentos nos últimos anos e o aumento das taxas de cura para muitos tipos de câncer da infância ${ }^{1,2,13}$. Dados nacionais apresentam taxa estimada de sobrevida de $64 \%$ para o 
câncer infantojuvenil (entre zero e 19 anos) ${ }^{3}$ e 75\% na Região Sul do país ${ }^{3}$. O presente estudo, realizado na mesma Região, demonstrou sobrevida global de 70,3\% (Gráfico 1) e livre de doença de 71,63\% (Gráfico 2). Esta última pode ser relacionada ao alto índice de recidivas encontrado. Espera-se contribuir para o delineamento de futuros estudos nessa localidade e para os Registros de Câncer de Base Populacional (RCBP) no Brasil.

Sabe-se que o estágio da doença no momento do diagnóstico $^{22}$, a presença de metástase e recidivas ${ }^{1,13,22}$ podem influenciar a sobrevida. A associação significativa entre o estadiamento clínico dos tumores sólidos e a situação atual do paciente reafirma que o avanço da doença reduz as chances de cura e aumenta a frequência de sequelas por exigir tratamento mais agressivo9. Contudo, é possível que vieses de informação tenham interferido nesses resultados, principalmente porque o estadiamento dos cânceres está em constante aprimoramento. A metástase (determinante na mortalidade dos pacientes) ${ }^{13,22}$ e as recidivas (que afetam sobremaneira a sobrevida e a qualidade de vida deles) ${ }^{1}$ também foram significativamente associadas com a situação do paciente. A não associação entre recidivas e estadiamento clínico, por outro lado, pode ser explicada pelo número reduzido da amostra.

Ressalta-se que, embora a origem dos dados analisados na presente pesquisa possa ter atuado como fator limitador, os resultados apresentados podem subsidiar as atividades no próprio $\mathrm{Hospital}^{3}$, uma vez que o corpo científico terá um panorama temporal e regional da população assistida. Por fim, levando em conta a falta de informaçóes em muitos registros, sugere-se a criação de protocolos unificados para o preenchimento dos prontuários para facilitar a coleta das informaçôes e contribuir com a condução de outros estudos, não somente no Hospital pesquisado, mas nos demais centros de assistência do país.

\section{CONCLUSÃO}

Os pacientes analisados na presente pesquisa eram na maioria leucêmicos, com maior frequência para o sexo masculino e faixa etária de 1-4 anos. E a sobrevida global e a livre de doença foram, respectivamente, de 70,3\% e $71,63 \%$.

\section{CONTRIBUIÇÕES}

Todos os autores contribuíram substancialmente na concepção ou no planejamento do estudo; na obtenção, análise e/ou interpretação dos dados; na redação e revisão crítica; e aprovaram a versão final a ser publicada.

\section{AGRADECIMENTOS}

Ao Dr. Mauro Carlos Agner Busato pela colaboração na escolha do teste estatístico.

\section{DECLARAÇÃO DE CONFLITO DE INTERESSES}

Nada a declarar.

\section{FONTES DE FINANCIAMENTO}

Coordenação de Aperfeiçoamento de Pessoal de Nível Superior (Capes). Código de Financiamento 001.

\section{REFERÊNCIAS}

1. Grabow D, Kaiser M, Hjorth L, et al. The PanCareSurFup cohort of 83,333 five-year survivors of childhood cancer: a cohort from 12 European countries. Eur J Epidemiol. 2018;33(3):335-49. doi: https://doi.org/10.1007/ s10654-018-0370-3

2. Gröbner SN, Worst BC, Weischenfeldt J, et al. The landscape of genomic alterations across childhood cancers. Nature. 2018;555(7696):321-7. doi: https:// doi.org/10.1038/nature25480

3. Instituto Nacional de Câncer José Alencar Gomes da Silva. Incidência, mortalidade e morbidade hospitalar por câncer em crianças, adolescentes e adultos jovens no Brasil: informaçóes dos registros de câncer e do sistema de mortalidade [Internet]. Rio de Janeiro: INCA; 2016 [acesso 2019 jun 17]. Disponível em: http://www1.inca. gov.br/wcm/incidencia/2017/pdf/versao-completa.pdf

4. Instituto Nacional de Câncer José Alencar Gomes da Silva [Internet]. Rio de Janeiro: INCA; [data desconhecida]. Tipos de câncer: câncer infantojuvenil; [modificado 2021 mar 4; acesso 2019 dez 10]. Disponível em: https://www. inca.gov.br/tipos-de-cancer/cancer-infantojuvenil

5. Stefan C, Bray F, Ferlay J, et al. Cancer of childhood in sub-Saharan Africa. Ecancermedicalscience. 2017;11:755. doi: https://doi.org/10.3332/ecancer.2017.755

6. Little J. Epidemiology of childhood cancer. Lyon, FR: International Agency for Research on Cancer; 1999. (IARC Scientific Publication; no 149).

7. Silva DB, Pires MMS, Nassar SM. Câncer pediátrico: análise de um registro hospitalar. J Pediatr (Rio J). 2002;78(5):409-14. doi: http://doi.org/10.1590/S002175572002000500012

8. Steliarova-Foucher E, Stiller C, Lacour B, et al. International Classification of Childhood Cancer, third edition. Cancer. 2005;103(7):1457-67. doi: https://doi. org/10.1002/cncr.20910

9. Zouain-Figueiredo GP, Zandonade E, Amorim MHC, et al. Perfil epidemiológico dos casos novos de câncer infanto-juvenil em hospital de referência no Espírito 
Santo, Brasil, de 1986 a 2010. Rev Bras Pesqui Saúde. 2016;17(4):109-20. doi: https://doi.org/10.21722/rbps. v17i4.14337

10. Reis RS, Santos MO, Thuler LCS. Incidência de tumores pediátricos no Brasil. Rev Bras Cancerol. 2007;53(1):5-15. doi: https://doi.org/10.32635/21769745.RBC.2007v53n1.1823

11. Diniz AB, Regis CA, Brito NP, et al. Perfil epidemiológico do câncer infantil em população atendida por uma unidade de oncologia pediátrica em Salvador-Bahia. Rev Ciênc Méd Biol. 2005;4(2):131-9. doi: http://doi. org/10.9771/cmbio.v4i2.4185

12. Mutti CF, Cruz VG, Santos LF, et al. Perfil clínico-epidemiológico de crianças e adolescentes com câncer em um serviço de oncologia. Rev Bras Cancerol. 2018;64(3):293-300. doi: https://doi. org/10.32635/2176-9745.RBC.2018v64n3.26

13. Ward E, DeSantis C, Robbins A, et al. Childhood and adolescent cancer statistics, 2014. CA Cancer J Clin. 2014;64(2):83-103. doi: http://doi.org/10.3322/ caac. 21219

14. Wiangnon S, Jetsrisuparb A, Komvilaisak P, et al. Childhood cancer incidence and survival 1985-2009, Khon Kaen, Thailand. Asian Pac J Cancer Prev. 2014;15(18):7989-93. doi: http://doi.org/10.7314/ apjcp.2014.15.18.7989

15. Ortega-García J, López-Hernández FA, Cárceles-Álvarez A, et al. [Analysis of small areas of paediatric cancer in the municipality of Murcia (Spain)]. An Pediatr (Barc). 2016;84(3):154-62. Spanish doi: http://doi. org/10.1016/j.anpede.2015.04.012

16. Ishihara H, Ohno Y, Fujii M, et al. Epidemiological analysis of childhood cancer in Japan based on population-based cancer registries, 1993-2009. Jpn J Clin Oncol. 2017;47(7):660-3. doi: https://doi.org/10.1093/ jjco/hyx041

17.Cidades@: sistema agregador de informaçóes sobre os municípios e estados do Brasil [Internet]. Version 4.4.13. Rio de Janeiro: IBGE. c2017. PNADC - Pesquisa Nacional por Amostra de Domicílios Contínua; 2018 [acesso 2019 jul 10]. Disponível em: https://cidades. ibge.gov.br/brasil/pr/panorama

18. Conselho Nacional de Saúde (BR). Resolução no 466, de 12 de dezembro de 2012. Aprova as diretrizes e normas regulamentadoras de pesquisas envolvendo seres humanos. Diário Oficial da União, Brasília, DF. 2013 jun 13; Seção 1:59.

19. Strahm B, Malkin D. Hereditary cancer predisposition in children: genetic basis and clinical implications. Int J Cancer. 2006;119(9):2001-6. doi: https://doi. org/10.1002/ijc.21962

20. American Cancer Society. Cancer facts \& figures 2019 [Internet]. Atlanta (GA): American Cancer Society; 2019 [cited 2019 June 30]. Available from: https://www. cancer.org/research/cancer-facts-statistics/all-cancerfacts-figures/cancer-facts-figures-2019.html

21. Peres P, Queiroz AMd, Moreira MR, et al. Odontopediatria aplicada ao câncer infantil: manifestaçôes clínicas e protocolo de atendimento. J Manag Prim Health Care. 2013;4(3):191-9. doi: https://doi.org/10.14295/jmphc. v4i3.188

22. Moreno F, Dussel V, Orellana L. Childhood cancer in Argentina: survival 2000-2007. Cancer Epidemiol. 2015;39(4):505-10. doi: https://doi.org/10.1016/j. canep.2015.04.010

Recebido em 3/9/2020

Aprovado em 19/2/2021 